\title{
Puerarin promotes rehabilitation of cartilage injury in juvenile rat model via inhibiting apoptosis: A mechanism study.
}

\author{
Chuangyi Zheng, Zhonglian Huang, Chunhua Peng, Zebin Ma, Yandan Zheng* \\ Department of Orthopedics, First Affiliated Hospital of Shantou University Medical College, Shantou, Guangdong, \\ PR China
}

\begin{abstract}
Objective: Perthes' disease is one of the most common pediatric surgical disease and affects children's health seriously. Pathogenesis of Perthes' disease is associated with pressure overload and inflammation. Puerarin was proved with promising efficacy on adult surgical disease. Our study is aimed explore effect of puerarin on cartilage injury of juvenile rat.

Methods: BALB/C rats were randomly assigned into three groups, including control group (A group), saline-treated group (B group) and puerarin-treated group (C group). Every group contains 10 rats. Saline and puerarin were treated with intragastric administration for $B$ group and $C$ group, respectively $(2.18 \mathrm{~g} / \mathrm{kg}$, twice a day, $4 \mathrm{w})$. Cartilage injury of juvenile rat model was established with Hulth method for $B$ group and $C$ group. Pathologic change of articular cartilage was assessed for three groups. Cellular activity was examined with MTT assays. Apoptosis was assessed with flow cytometry.

Results: Intact and smooth structure integrity of chondrocyte was observed in control group. Compared with saline-treated group, cartilage injury in puerarin-treated group was significantly alleviated, including reduced surface rupture, intact cell structure and relieving inflammation $(\mathbf{P}<0.05)$. Puerarin treatment significantly reduced the percentage of apoptosis and enhanced cell activity of chondrocyte $(\mathbf{P}<\mathbf{0 . 0 5})$.

Conclusions: Puerarin promotes rehabilitation of cartilage injury in juvenile rat model. Potential mechanism was that puerarin inhibited apoptosis and enhanced cell activity of chondrocyte.
\end{abstract}

Keywords: Chondrocyte, Apoptosis, Puerarin, Perthes' disease.

Accepted on October 26, 2017

\section{Introduction}

Perthes' disease is one of the most common degenerative joint disease disorder, which is characterized by dysfunction of hip joint $[1,2]$. Primary cause of Perthes' disease is still unclear, with recent evidences indicated that multiple factors were involved in pathogenesis of Perthes' disease, such as hereditary factor, perinatal joint damage, gene mutation and diet factors $[3,4]$. Latest research showed that pressure stimulated inflammatory cytokines secretion of chondrocytes and promoted degradation of collagen via activating proteolytic enzyme, which finally caused matrix denaturalization of chondrocytes [5]. In addition, auto repairing ability of chondrocytes is insufficient to reverse pathophysiology process of cartilage injury. Moreover, cartilage injury was tightly linked to the development of Perthes' disease, while current treatment for Perthes' disease cannot provide ideal efficacy on cartilage injury [6,7]. Chinese medicine has a special function on prevention and treatment of degenerative joint disease. Studies showed that puerarin, extracted from Chinese medicinal herb, delayed cell senescence, improved metabolism and inhibited degenerative change [8]. Thus, our study established cartilage injury in juvenile rat model to mimic
Perthes' disease, and explored effect of puerarin on rehabilitation of cartilage injury so as to provide theoretical basis for puerarin-related clinical treatment.

\section{Materials and Methods}

\section{Experimental animal}

$30 \mathrm{BALB} / \mathrm{C}$ male juvenile rats were purchased from laboratory animal center of Shantou University Medical College.

Rats were used for all experiments, and all procedures were approved by the Animal Ethics Committee of First Affiliated Hospital of Shantou University Medical College.

\section{Experimental agents}

Decoctum of puerarin was prepared by Chengdu University of TCM. Original medicine of puerarin was processed into puerarin decoctum with a concentration of $1 \mathrm{~g} / \mathrm{ml}$. Perform aseptic packaging of puerarin decoctum after filtration and high temperature sterilization. Store at $-4^{\circ} \mathrm{C}$. 


\section{Experiment instruments and equipment}

Table model high speed centrifuge (Ependorf), Clean bench (Suzhou Purification Equipment Co., China), Thermostatic Incubator (Shellab, USA), Inverted phase contrast microscope (Olympus, Japan) and Ultra cold storage freezer (Siemens, Germany).

\section{Drugs and reagents}

DMEM medium (Gibco, USA), Fetal bovine serum (Hangzhou sijiqing biological products co., LTD., China), Tyrisin (Sigma, USA), Collagenase typeII (Gibco, USA), MTT kits (Hangzhou blue sky, LTD., China), Goat anti-rat monoclonal antibody of collagenase type II (boster biological engineering co., LTD., China), SABC kits (boster biological engineering co., LTD., China) and Annexin V FITC apoptosis assays kit (boster biological engineering co., LTD., China).

\section{Experiment grouping}

$\mathrm{BALB} / \mathrm{C}$ rats were randomly assigned into three groups, including control group (Control group), saline-treated group (Saline-treated group) and puerarin-treated group (Puerarintreated group). Every group contains 10 rats. Saline and puerarin were treated with intragastric administration for Saline-treated group and Puerarin-treated group, respectively $(2.18 \mathrm{~g} / \mathrm{kg}$, twice a day, $4 \mathrm{w})$.

\section{Animal model}

Model of cartilage injury was established in juvenile rats with Hulth improved method. Protocols are as follows: bilateralis buttocks were sterilized for operation. Hip articular capsules were opened via bilateral hip joint lateral operation approach. Articular surfaces were removed completely. Cover sterilized dressing upon the operating position and change dressings regularly. All operated rats received constraint-induced movement for $30 \mathrm{~min}$ a day after operation.

\section{Experimental sampling}

All rats received euthanasia after 4 w intragastric administration. Remove rat body into clean bench after $10 \mathrm{~min}$ soak in $75 \%$ alcohol. Cut the top of femoral head cartilage with $2 \mathrm{~mm}$ thickness. Digest tissues of femoral head cartilage with $0.25 \%$ trypsin. Add $5 \mathrm{ml} 0.25 \%$ collagenase typeII and maintain digestion for 4 to $6 \mathrm{~h}$ in the incubator. Add $5 \mathrm{ml}$ fetal bovine serum to terminate digestion after most chondrocytes were separated. Culture chondrocytes in the incubator for puerarin treatment.

\section{MTT assays}

Treatment was performed as chondrocytes were cultured into the log phase. Incubate cells into a 96-well plate. Culture chondrocytes in the incubator overnight. Every well was added into $100 \mathrm{ml}$ puerarin solution with different concentration. MTT working solution was added with routine protocols.
Discard supernatant and add $150 \mu$ DMSO. Absorbance value was assessed with the microplate reader.

\section{Flow cytometry}

Prepared cell suspension solution of chondrocyte for flow cytometry. Incubate chondrocytes into a 96-well plate with cell density of $2 \times 10^{5}$. Every well was added into $100 \mathrm{ml}$ puerarin solution with different concentration. Culture chondrocytes in the incubator for $24 \mathrm{~h}$. Resuspend chondrocytes and add $250 \mu \mathrm{l}$ buffer solution to adjust cell density $\left(1 \times 10^{6}\right)$. Add $5 \mu \mathrm{l}$ Annexin V FITC and PI solution. Maintain reaction away from light for $15 \mathrm{~min}$. Flow cytometry was performed with routine protocols.

\section{Statistical analysis}

SPSS16.0 software was used for data processing. Measurement data are normal distribution to $\overline{\mathrm{x}} \pm \mathrm{S}$. T-test was performed for statistical significance. $\mathrm{P}$ value $<0.05$ was considered to be statistically significant.

\section{Results}

\section{Puerarin improved post-injury morphology of chondrocytes}

Intact and smooth structure integrity of chondrocytes was observed in control group, while irregularity and structure of chondrocytes were compromised in saline-treated group. Moreover, HE staining showed that maldistribution of chondrocytes and vague tidal line in post-injury cartilage tissues (Figure 1). Compared with saline-treated group, cartilage injury in puerarin-treated group was significantly alleviated (Figure 1). Mankin's score of puerarin-treated group was significantly higher than saline-treated group $(\mathrm{P}<0.05$, Table 1).

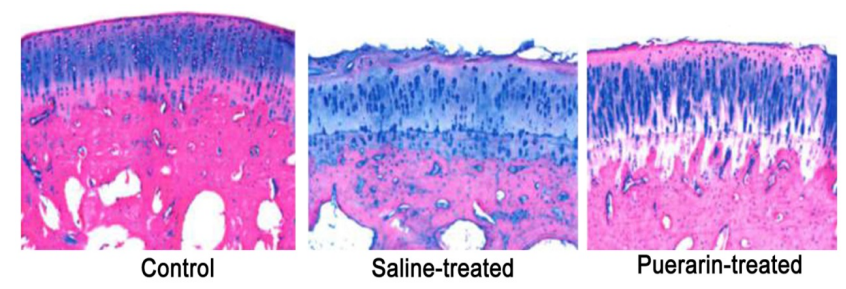

Figure 1. Pathological change of chondrocytes in three groups (HE staining, X100).

\section{Puerarin enhanced cell activity of chondrocytes}

Absorbance values of three groups were $0.75 \pm 0.01$ (Control group), $0.07 \pm 0.02$ (Saline-treated group) and $0.10 \pm 0.01$ (Puerarin-treated group), verified by MTT assays. Compared with control group, absorbance values of saline-treated group and puerarin-treated group were significantly increased $(\mathrm{P}<0.05$, Figure 2$)$, suggesting puerarin enhanced cell activity of chondrocytes after cartilage injury. 


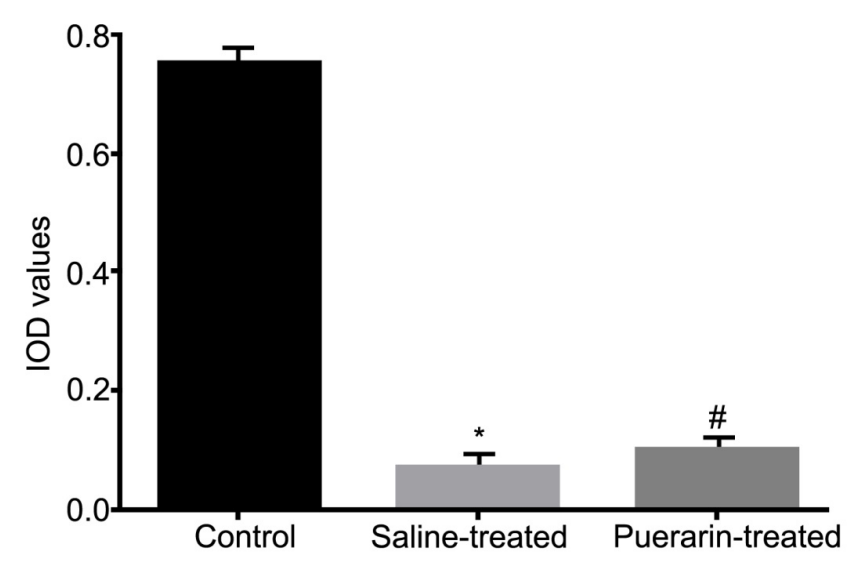

Figure 2. Analysis of absorbance values in three groups. ${ }^{*} P<0.05$, versus control group. ${ }^{\#} P<0.05$, versus control group.

\section{Puerarin inhibited apoptosis of chondrocytes after cartilage injury}

Flow cytometry showed that the apoptosis rate of chondrocytes was $9.70 \%$ (Figure $3 \mathrm{~A}$ ) in control group, and $21.87 \%$ in salinetreated group (Figure 3B). Compared with saline treatment, puerarin significantly decreased the apoptosis rate to $10.55 \%$ $(\mathrm{P}<0.05$, Figure 3C), suggesting that puerarin inhibited apoptosis of chondrocytes.
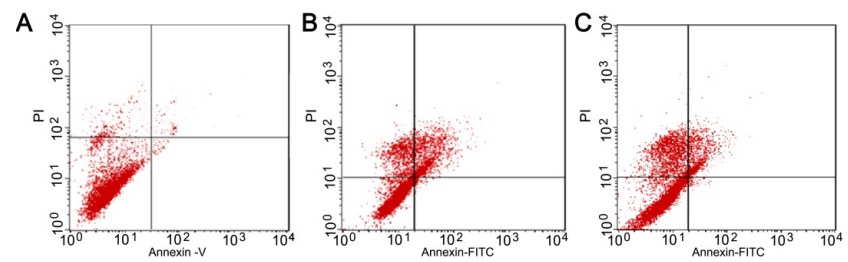

Figure 3. Apoptosis rates in three groups.

Table 1. Analysis of Mankin's score in three groups.

\begin{tabular}{lll}
\hline Group & $\mathbf{n}$ & Mankin's score \\
\hline Control & 10 & $0.75 \pm 0.05$ \\
\hline Saline-treated & 10 & $7.57 \pm 1.55^{*}$ \\
\hline Puerarin-treated & 10 & $5.15 \pm 1.00^{\#}$ \\
\hline${ }^{*} \mathrm{P}<0.05$, versus control group. ${ }^{\#} \mathrm{P}<0.05$, versus control group. \\
\hline
\end{tabular}

\section{Discussion}

Articular cartilage injury is one of the most important causes in the progression of Perthes' disease. Mechanisms underlying cartilage injury are still controversial. Recent studies further demonstrated both pressure overload and inflammation enhanced apoptosis of chondrocytes to cause poor prognosis of Perthes' disease, including function impairment, worsen pain and exacerbated hydrarthrosis $[9,10]$. However, the apoptosis of chondrocytes can't be reversed itself under the condition of Perthes' disease. As the only cellular constituent, physiological and pathological changes of chondrocytes are tightly correlated with onset and progression of Perthes' disease [11].
We used Hulth improved method to establish the model of cartilage injury in juvenile rats. Rats were forced to complete physical activity after facies lunata was removed. This model mimics pressure overload and inflammation in Perthes' disease. Moreover, this experimental design was more in line with potential mechanisms of Perthes' disease.

Puerarin, also known as 7, 4'-dihydroxy-8-Cglucosylisoflavone $\left(\mathrm{C}_{21} \mathrm{H}_{20} \mathrm{O}_{10}\right)$, is macromolecular compound which was harvested after separation and purification of Radix Puerariae. Modern pharmacological research showed that puerarin inhibited pulmonary fibrosis via reducing the content of NO in lung tissue, and decreased spontaneous or dexamethasone-induced apoptosis of thymocytes [12,13]. A certain concentration of puerarin has anti-inflammatory properties via inhibiting proliferation of vascular endothelial cell and leukocyte adhesion $[14,15]$. In addition, puerarin was proved with protecting function on Lysosomes and mitochondria via regulating mitochondrion membrane potential $[16,17]$. Latest study showed that low concentration of puerarin could activate osteoblast and inhibit proliferation and differentiation of osteoclast [18-20]. However, there is no report about effect of puerarin on chondrocyte.

Our study firstly explored direct effect of puerarin on chondrocyte in Perthes' disease. Pressure and inflammation indeed caused cartilage injury in juvenile rat model. What's more, puerarin alleviated cartilage injury via enhancing cell activity and inhibiting apoptosis of chondrocytes, verified by MTT and flow cytometry. Cartilage is composed of chondrocytes and extracellular matrix. The structure of cartilage had four layers from the inside-out, including superficial layer, transition layer, radiation layer and calcified layer. Our study showed that cartilage injury resulted in maldistribution of chondrocytes and vague tidal line, suggesting cartilage was indeed impaired. Puerarin treatment inhibited apoptosis of chondrocytes and reversed injury progression, suggesting puerarin had promising efficacy on cartilage injury.

In summary, pressure and inflammation inhibit proliferation of chondrocytes and enhanced apoptosis. Puerarin alleviates cartilage injury via inhibiting apoptosis and enhanced cell activity of chondrocyte.

\section{Conclusion}

Puerarin promotes rehabilitation of cartilage injury in pressure or inflammation induced Perthes' disease. Potential mechanism was that puerarin inhibited apoptosis and enhanced cell activity of chondrocyte.

\section{References}

1. Sen ES, Clarke SL, Ramanan AV. The child with joint pain in primary care. Best Pract Res Clin Rheumatol 2014; 28 : 888-906.

2. Chambers HG. Update on neuromuscular disorders in pediatric orthopaedics: Duchenne muscular dystrophy, 
myelomeningocele, and cerebral palsy. J Pediatr Orthop 2014; 34: 44-48.

3. Mazloumi SM, Ebrahimzadeh MH, Kachooei AR. Evolution in diagnosis and treatment of Legg-CalvePerthes disease. Arch Bone Jt Surg 2014; 2: 86-92.

4. Chaudhry S, Phillips D, Feldman D. Legg-Calve-Perthes disease: an overview with recent literature. Bull Hosp Jt Dis 2014; 72: 18-27.

5. Johnson ZI, Shapiro IM, Risbud MV. Extracellular osmolarity regulates matrix homeostasis in the intervertebral disc and articularcartilage: evolving role of TonEBP. Matrix Biol 2014; 40: 10-16.

6. Demoor M, Ollitrault D, Gomez-Leduc T, Bouyoucef M, Hervieu M, Fabre H, Lafont J, Denoix JM, Audigié F, Mallein-Gerin F, Legendre F, Galera P. Cartilage tissue engineering: molecular control of chondrocyte differentiation for proper cartilagematrix reconstruction. Biochim Biophys Acta 2014; 1840: 2414-2440.

7. Oconor CJ, Case N, Guilak F. Mechanical regulation of chondrogenesis. Stem Cell Res Ther 2013; 4: 61.

8. Mohammadi H, Mequanint K, Herzog W. Computational aspects in mechanical modeling of the articular cartilage tissue. Proc Inst Mech Eng H 2013; 227: 402-420.

9. Mabvuure N, Hindocha S, Khan WS. The role of bioreactors in cartilage tissue engineering. Curr Stem Cell Res Ther 2012; 7: 287-292.

10. Galle J, Bader A, Hepp P, Grill W, Fuchs B, Käs JA, Krinner A, Marquass B, Muller K, Schiller J, Schulz RM, von Buttlar M, von der Burg E, Zscharnack M, Löffler M. Mesenchymal stem cells in cartilage repair: state of the art and methods to monitor cell growth, differentiation and cartilage regeneration. Curr Med Chem 2010; 17: 2274-2291.

11. Bao JP, Chen WP, Wu LD. Lubricin: a novel potential biotherapeutic approaches for the treatment of osteoarthritis. Mol Biol Rep 2011; 38: 2879-2885.

12. Mardones R, Larrain C. Cartilage restoration technique of the hip. J Hip Preserv Surg 2015; 3: 30-36.

13. Nazempour A, Van Wie BJ. Chondrocytes, mesenchymal stem cells, and their combination in articular cartilage regenerative medicine. Ann Biomed Eng 2016; 44: 1325-1354.

14. Matta C, Fodor J, Csernoch L, Zakany R. Purinergic signalling-evoked intracellular $\mathrm{Ca}(2+)$ concentration changes in the regulation of chondrogenesis and skeletal muscle formation. Cell Calcium 2016; 59: 108-116.

15. Murray SS, Brochmann Murray EJ, Wang JC, Duarte ME. The history and histology of bone morphogenetic protein. Histol Histopathol 2016; 31: 721-732.

16. Longo L, Greco A, Rea A, Lo Vasco VR, De Virgilio A. Relapsing polychondritis: A clinical update. Autoimmun Rev 2016; 15: 539-543.

17. Hartmann K, Koenen M, Schauer S, Wittig-Blaich S, Ahmad M, Baschant U, Tuckermann JP. Molecular actions of glucocorticoids in cartilage and bone during health, disease, and steroid therapy. Physiol Rev 2016; 96: 409-447.

18. Scarfî S. Use of bone morphogenetic proteins in mesenchymal stem cell stimulation of cartilage and bone repair. World J Stem Cells 2016; 8: 1-12.

19. Panadero JA, Lanceros-Mendez S, Ribelles JL. Differentiation of mesenchymal stem cells for cartilage tissue engineering: Individual and synergetic effects of three-dimensional environment and mechanical loading. Acta Biomater 2016; 33: 1-12.

20. Pan Z, Duan P, Liu X, Wang H, Cao L, He Y, Dong J, Ding J. Effect of porosities of bilayered porous scaffolds on spontaneous osteochondral repair in cartilagetissue engineering. Regen Biomater 2015; 2: 9-19.

\section{*Correspondence to}

Yadan Zheng

Department of Orthopedics

First Affiliated Hospital of Shantou University Medical College

PR China 